\title{
A study of power availability in Oleh community in Isoko South local Government area of Delta State, Nigeria
}

\author{
S.O. Otuagoma* \\ Nigeria \\ Received: 11-February-2016; Revised: 19-March-2016; Accepted: 22-March-2016 \\ (C)2016 ACCENTS
}

Department of Electrical/Electronic Engineering, Faculty of Engineering, Delta State University, Oleh Campus,

\begin{abstract}
In this study, the availability of electric power supply in the Oleh community of Isoko South Local Government Area of Delta State, Nigeria was carried out. The methods employed in achieving this include the use of Hour-Meter to register the number of hours supply was available for a period of three months, Multimeter and Ammeter were used to measure the supply voltage and current at any given time. The load shedding method adopted in the community by the Benin Electricity Distribution Company, BEDC was also investigated. The results showed that power availability in the community for the three months under review was very bad. The average percentage of power available for the three months was $11.8 \%$, meaning $88.2 \%$ of the time, power was unavailable. The power reliability index for each month of the study was negative; -5.94, -7.82 and -5.94 for June, July and August, 2015 respectively against a recommended value of high reliability of 0.989. This poor power availability accounts for the underdevelopment of the community.
\end{abstract}

Keywords

Electric power, Voltage, Hour-meter, Load shedding and Reliability.

\section{Introduction}

The demand for electric energy worldwide is on the increase. This is so because electric power is fundamental of human activities since all human activities depend on one form of energy or another. Electrical energy is the most important catalyst for economic development of any country [1]. In-spite of this supreme importance of electric energy to socioeconomic development, Nigeria as a country is lacking adequate supply of electric power even though the country is endowed with numerous energy resources. Out of the nation's 170 million people, only about $40 \%$ of the population that have access to grid connected electricity and it is even worse in the rural areas [1]. Table 1 shows the status of energy sector in Nigeria compared to other countries in the Sub-Sahara Africa (SSA) region. It can be seen from Table 1 that only $26 \%$ of the population that have access to electricity in the rural areas of the country and according to World Energy Outlook 2012, 1.3 billion people worldwide still lack access electricity while 2.7 billion rely solely on traditional biomass to meet their energy needs.

\footnotetext{
*Author for correspondence
}

The United Nations estimated that among those with access to electricity, 1 billion people have poor quality electricity or can only obtain it intermittently from unreliable grid networks [2]. This is especially so in the case of emerging economies like Nigeria, where over $50 \%$ of the population live in rural communities without electrical power [3].

The inadequacy in power generation in Nigeria has resulted in high System Average Interruption Duration Index (SAIDI). System Average Interruption Duration Index is one of the Distribution System Reliability (DSR) indices used in the assessment of distribution performance. As presented in Table 2, the reliability index in Nigeria as compared to other selected countries in the world is embarrassingly high. The Manufacturers' Association of Nigeria (MAN) reported Nigeria's SAIDI of 60,000 minutes/annum or greater than, seems to be more realistic as it agreed with a Figure of 87,639 minutes/annum obtained in another independent research [5]. This high SAIDI has resulted in poor per capita electricity consumption, which in turn has greatly affected the nation's economic growth especially in the rural areas. 
Table 1 Key indicators and electrification rates in selected SSA Countries [4]

\begin{tabular}{|c|c|c|c|c|c|c|c|c|}
\hline Country & $\begin{array}{l}\text { Populati } \\
\text { on } \\
\text { (Million) }\end{array}$ & $\begin{array}{l}\text { Total Area } \\
\left(\mathrm{Km}^{2}\right)\end{array}$ & $\begin{array}{l}\text { Density } \\
\text { (Person } \\
\text { Per } \\
\mathrm{Km}^{2} \text { ) }\end{array}$ & $\begin{array}{l}\text { Gni } \\
\text { (Us\$ Per } \\
\text { Capital) }\end{array}$ & $\begin{array}{l}\text { Share of } \\
\text { Population } \\
\text { Below } \\
\text { Poverty } \\
\text { Line }\end{array}$ & $\begin{array}{l}\text { Electrificati } \\
\text { on } \\
\text { (National) }\end{array}$ & $\begin{array}{l}\text { Electrificatio } \\
\text { n (Rural) }\end{array}$ & $\begin{array}{l}\text { Power } \\
\text { Consu } \\
\text { mption } \\
\text { (Kwh } \\
\text { Per } \\
\text { Capital) }\end{array}$ \\
\hline Ethiopia & 80 & $1,104,000$ & 72 & 280 & $44.2 \%$ & $15.3 \%$ & $2.0 \%$ & 40 \\
\hline Kenya & 39 & 580,000 & 67 & 730 & $47 \%$ & $15 \%$ & $5.0 \%$ & 151 \\
\hline Mozambique & 22 & 800,000 & 27.5 & 380 & $55 \%$ & $11.7 \%$ & $6.3 \%$ & 472 \\
\hline Nigeria & 170 & 924,000 & 184 & 1,170 & $34.1 \%$ & $46.8 \%$ & $26 \%$ & 137 \\
\hline Rwanda & 10 & 26,340 & 380 & 440 & $56.9 \%$ & $4.8 \%$ & $1.3 \%$ & 20 \\
\hline South Africa & 48 & $1,221,000$ & 40 & 5,820 & $22 \%$ & $75 \%$ & $55 \%$ & 4986 \\
\hline
\end{tabular}

Table 2 SAIDI for some Countries [5]

\begin{tabular}{ll}
\hline Country & $\begin{array}{l}\text { Saidi } \\
\text { (Minutes/Annum) }\end{array}$ \\
\hline France & 52 \\
Singapore & $\approx 1.5$ \\
USA & 88 \\
Nigeria (PHCN) & 900 \\
Nigeria (MAN Study) & $\geq 60,000$ \\
Nigeria(Ogujor and Orobor,2010) & 87,639 \\
\hline
\end{tabular}

In this study, the availability of electric power supply to Oleh in Delta State, Nigeria was carried out The study area, is situated in the South-Western part of Delta State between latitudes $5^{0} 27^{\prime} 37.2$ ' $\mathrm{N}$ and longitudes $6^{0} 12^{\prime} 02.2^{\prime}$ 'E (Figure 1). It is the Head Quarter of Isoko South Local Government Area of the state. It is among the oil producing communities in Delta State with oil wells located at different places in the community. It also plays host to the Shell Petroleum Development Company, SPDC and two faculties of the Delta State University, Abraka the Faculty of Engineering and Law where one oil well is located.

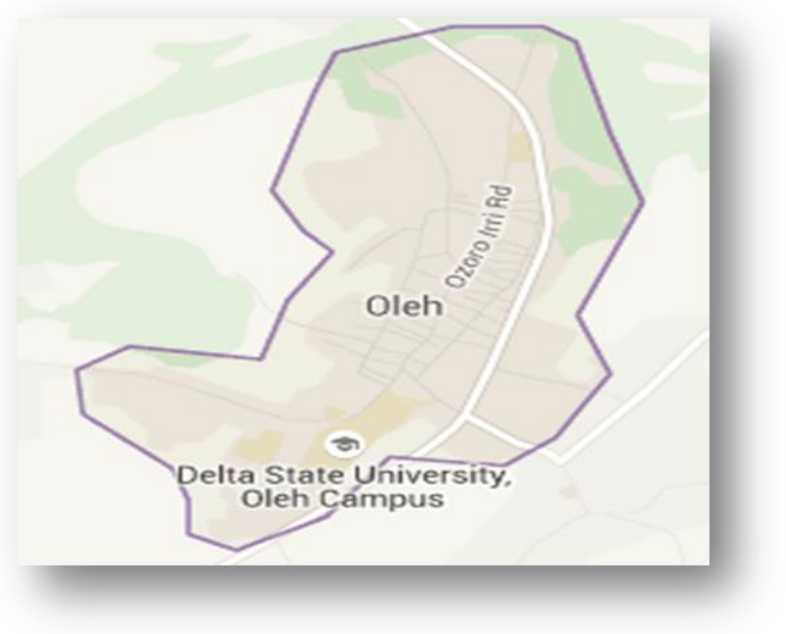

Figure 1 Map of Oleh in Isoko, Delta State, Nigeria
With the presence of these two faculties, especially the faculty of Engineering where heavy equipment is installed, such as electric welding machines, electric furnace, heavy duty lathe machines and other laboratory equipments, there is greater need for constant electric power supply to the community to meet the need of both students and staff of the campus and the general populace. Oleh is endowed with abundance of crude oil and gas as part of its natural resources and most of the gas is flowing to waste through gas flaring instead of converting it to a source of power generation. In-spite of the abundance of resources, the community suffers a lot from frequent power unavailability and this is really detrimental the faculty of Engineering where heavy demand of electric energy is needed. The study therefore is to investigate how bad the situation of power unavailability is in the community.

\section{Materials and methods}

To ascertain electric power availability in Oleh, the following measurement procedures were adopted. (1) measurement of the number of hours in a day that supply was available using the hour-meter, (2) measurement of the supply voltage and current using multi-meter, (3) investigating the load shedding arrangement by the supply authority to ascertain how many hours in a day the community is supposed to receive supplies from the generating station at Ughelli and (4) to determine the reliability index of the power supply system.

\subsection{Determination of the availability of supply using hour-meter}

The hour-meter is an instrument that tracks and records elapsed time, normally displayed in hours and minutes. The majority of the Hour-meters are used to log running time of equipment to assure proper maintenance of expensive machines or systems. 


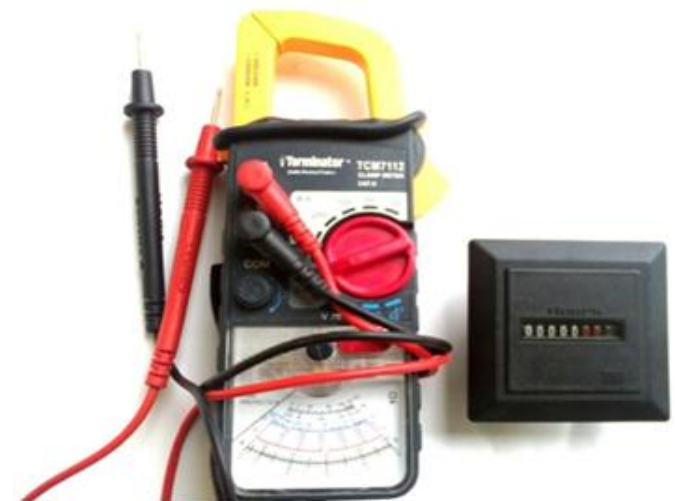

Figure 2 (a) Arm-probe (b) Hour-meter

The Hour-meter used in this study is the AC $220-$ $240 \mathrm{~V}, 50 \mathrm{H}_{\mathrm{z}}$ electric 7 digits display Hour-meter. It was installed in two different locations with different operating load shedding schedule. One was installed in the campus at the Faculty of Law substation and the other at the Emiye substation of Ogemudia road Oleh. The Hour-meter records the number of hours electrical power is available to any substation or accommodation. It can be read daily or monthly and it counts continuously as long as there is power supply to it with the red calibration indicating the minutes and the white indicating the hour of supply. Figure 2 shows the measuring instruments used in this study. The meter was read daily and the actual reading of the day was determined by using equation 1.

$A_{t}=C_{t}-P_{t}$

Where $A_{t}$ is the actual time in an hour

$\mathrm{P}_{\mathrm{t}}$ is the previous time in an hour

$\mathrm{C}_{\mathrm{t}}$ is the present time in an hour.

\subsection{Voltage and current measurements}

In this study, voltage and current measurements are crucial for the determination of available power for a given period of time. Figure $2 a$ shows the instrument used to measure the voltage and current from the supply authority and this was done any time there was supply. The amount of power available was determined using equation 2 .

$P_{e}=I V \cos \phi$

Where $\mathrm{P}_{\mathrm{e}}$ is electrical power

I is supplied current

$\mathrm{V}$ is the supplied voltage

$\cos \Phi$ is the power factor, p.f

The voltage and current were monitored for a period of three months and the data collected were recorded.
The average values of voltage and current with the number of hours' supply were available from the network were used to give an appropriate estimate for consumer bills instead of the flat rate estimation of bills by BEDC which is usually too high. The equation 3 was used for the calculation of the number of units consumed in $\mathrm{kWh}$ per month.

$$
E=P_{e} t
$$

Where $\mathrm{E}$ is the total energy consumed in $\mathrm{kWh}$ $\mathrm{P}_{\mathrm{e}}$ is electrical Power $\mathrm{t}$ is time power was available.

\subsection{Load shedding method}

In a deregulated environment like Nigeria, voltage instability is a growing problem, since most of the systems are interconnected and are always operating at near stability limit as its operating point changes continually. This results in a rapid voltage drop leading to system collapse [6] a term frequently used by energy providers in Nigeria. Load shedding is a deliberate action taken by the energy provider during emergency to ensure system stability by curtailing system load. It is an effective method of alleviating the problem of system collapse. When the energy provider receives more demand for electrical power than its generating or transmission or installed capacity can deliver, the provider has to resort to rationing of the available electricity to its customers [7].

Typically, load shedding protects the system against excessive frequency or voltage decline by attempting to balance real and reactive power supply and demand in the system [8]. The load shedding curtails amount of load in the power system until the available generation improves to meet up with the required demand. The amount of load to be shed is determined by the energy provider at the centralised load centres and the decision communicated to local or sub-load centres where it will be implemented [9]. In most cases, load shedding does not favour the rural areas since they are the first to be load shedded in times of electric power instability. The Tables show the load shedding rosters to Isoko (Oleh) from the transmission sub-station at Ughelli in Delta State, Nigeria in the months of June, July and August, 2015. Table 4 shows the load shedding roster in July, 2015 at 60MVA, $132 \mathrm{KV} / 33 \mathrm{KV}$ transformer $\mathrm{T} 1$. Table 5 shows the load shedding rosters in August, 2015 at $60 \mathrm{MVA}, 132 \mathrm{KV} / 33 \mathrm{KV}$ transformer T1. 
Table 3 Load shedding roster in June, 2015 at 60MVA,132KV/33KV transformer T1

\begin{tabular}{|c|c|c|c|c|c|}
\hline \multirow[t]{2}{*}{ Date } & \multirow[t]{2}{*}{ Day } & \multicolumn{4}{|c|}{ Period of the day } \\
\hline & & 06:00-10:00 & 10:00-02:00 & 02:00-06:00 & 06:00-06:00 \\
\hline $01 / 06 / 2015$ & MONDAY & A & B & $\mathrm{C}$ & A \\
\hline $02 / 06 / 2015$ & TUESDAY & B & $\mathrm{C}$ & A & B \\
\hline $03 / 06 / 2015$ & WEDNESDAY & $\mathrm{C}$ & A & B & $\mathrm{C}$ \\
\hline $04 / 06 / 2015$ & THURSDAY & A & B & $\mathrm{C}$ & A \\
\hline $05 / 06 / 2015$ & FRIDAY & B & $\mathrm{C}$ & A & B \\
\hline 06/06/2015 & SATURDAY & $\mathrm{C}$ & A & B & $\mathrm{C}$ \\
\hline $07 / 06 / 2015$ & SUNDAY & A & B & $\mathrm{C}$ & A \\
\hline 08/06/2015 & MONDAY & B & $\mathrm{C}$ & A & B \\
\hline 09/06/2015 & TUESDAY & $\mathrm{C}$ & A & B & $\mathrm{C}$ \\
\hline $10 / 06 / 2015$ & WEDNESDAY & A & B & $\mathrm{C}$ & A \\
\hline $11 / 06 / 2015$ & THURSDAY & B & $\mathrm{C}$ & A & B \\
\hline $12 / 06 / 2015$ & FRIDAY & $\mathrm{C}$ & A & B & $\mathrm{C}$ \\
\hline $13 / 06 / 2015$ & SATURDAY & A & B & $\mathrm{C}$ & A \\
\hline $14 / 06 / 2015$ & SUNDAY & B & $\mathrm{C}$ & A & B \\
\hline $15 / 06 / 2015$ & MONDAY & $\mathrm{C}$ & A & B & $\mathrm{C}$ \\
\hline $16 / 06 / 2015$ & TUESDAY & A & B & $\mathrm{C}$ & A \\
\hline $17 / 06 / 2015$ & WEDNESDAY & B & $\mathrm{C}$ & A & B \\
\hline $18 / 06 / 2015$ & THURSDAY & $\mathrm{C}$ & A & B & $\mathrm{C}$ \\
\hline $19 / 06 / 2015$ & FRIDAY & A & B & $\mathrm{C}$ & A \\
\hline $20 / 06 / 2015$ & SATURDAY & B & $\mathrm{C}$ & A & B \\
\hline $21 / 06 / 2015$ & SUNDAY & $\mathrm{C}$ & A & B & $\mathrm{C}$ \\
\hline $22 / 06 / 2015$ & MONDAY & A & B & $\mathrm{C}$ & A \\
\hline $23 / 06 / 2015$ & TUESDAY & B & $\mathrm{C}$ & A & B \\
\hline $24 / 06 / 2015$ & WEDNESDAY & $\mathrm{C}$ & A & B & $\mathrm{C}$ \\
\hline $25 / 06 / 2015$ & THURSDAY & A & B & $\mathrm{C}$ & A \\
\hline $26 / 06 / 2015$ & FRIDAY & B & $\mathrm{C}$ & A & B \\
\hline $27 / 06 / 2015$ & SATURDAY & $\mathrm{C}$ & A & B & $\mathrm{C}$ \\
\hline $28 / 06 / 2015$ & SUNDAY & A & B & $\mathrm{C}$ & A \\
\hline $29 / 06 / 2015$ & MONDAY & B & $\mathrm{C}$ & A & B \\
\hline $30 / 06 / 2015$ & TUESDAY & $\mathrm{C}$ & A & B & $\mathrm{C}$ \\
\hline
\end{tabular}

$\mathrm{A}=$ Agbarho/Eku, $\mathrm{B}=$ Aladja, $\mathrm{C}=\mathrm{Isoko} / \mathrm{Kwale}$

Table 4 Load shedding roster in July, 2015 at 60MVA, 132KV/33KV transformer T1

\begin{tabular}{|c|c|c|c|c|c|}
\hline \multirow[t]{2}{*}{ Date } & \multirow[t]{2}{*}{ Day } & \multicolumn{4}{|c|}{ Period of the day } \\
\hline & & 06:00-10:00 & 10:00-02:00 & 02:00-06:00 & 06:00-06:00 \\
\hline $01 / 07 / 2015$ & WEDNESDAY & A & B & $\mathrm{C}$ & A \\
\hline $02 / 07 / 2015$ & THURSDAY & B & $\mathrm{C}$ & A & B \\
\hline 03/07/2015 & FRIDAY & $\mathrm{C}$ & A & B & $\mathrm{C}$ \\
\hline $04 / 07 / 2015$ & SATURDAY & A & B & $\mathrm{C}$ & A \\
\hline 05/07/2015 & SUNDAY & B & $\mathrm{C}$ & A & B \\
\hline $06 / 07 / 2015$ & MONDAY & $\mathrm{C}$ & A & B & $\mathrm{C}$ \\
\hline 07/07/2015 & TUESDAY & A & B & $\mathrm{C}$ & A \\
\hline 08/07/2015 & WEDNESDAY & B & $\mathrm{C}$ & A & B \\
\hline 09/07/2015 & THURSDAY & $\mathrm{C}$ & A & B & $\mathrm{C}$ \\
\hline $10 / 07 / 2015$ & FRIDAY & A & B & $\mathrm{C}$ & A \\
\hline $11 / 07 / 2015$ & SATURDAY & B & $\mathrm{C}$ & A & B \\
\hline $12 / 07 / 2015$ & SUNDAY & $\mathrm{C}$ & A & B & $\mathrm{C}$ \\
\hline $13 / 07 / 2015$ & MONDAY & A & B & $\mathrm{C}$ & A \\
\hline $14 / 07 / 2015$ & TUESDAY & B & $\mathrm{C}$ & A & B \\
\hline $15 / 07 / 2015$ & WEDNESDAY & $\mathrm{C}$ & A & B & $\mathrm{C}$ \\
\hline $16 / 07 / 2015$ & THURSDAY & A & B & $\mathrm{C}$ & A \\
\hline $17 / 07 / 2015$ & FRIDAY & B & $\mathrm{C}$ & A & B \\
\hline $18 / 07 / 2015$ & SATURDAY & $\mathrm{C}$ & A & B & $\mathrm{C}$ \\
\hline $19 / 07 / 2015$ & SUNDAY & A & B & $\mathrm{C}$ & A \\
\hline $20 / 07 / 2015$ & MONDAY & B & $\mathrm{C}$ & A & B \\
\hline $21 / 07 / 2015$ & TUESDAY & $\mathrm{C}$ & A & B & $\mathrm{C}$ \\
\hline
\end{tabular}


International Journal of Advanced Computer Research, Vol 6(23)

\begin{tabular}{cccccc}
\hline Date & Day & \multicolumn{5}{c}{ Period of the day } \\
\cline { 3 - 6 } & & $\mathbf{0 6 : 0 0 - 1 0 : 0 0}$ & $\mathbf{1 0 : 0 0 - 0 2 : 0 0}$ & $\mathbf{0 2 : 0 0 - 0 6 : 0 0}$ & $\mathbf{0 6 : 0 0 - 0 6 : 0 0}$ \\
\hline $22 / 07 / 2015$ & WEDNESDAY & $\mathrm{A}$ & $\mathrm{B}$ & $\mathrm{C}$ & $\mathrm{A}$ \\
$23 / 07 / 2015$ & THURSDAY & $\mathrm{B}$ & $\mathrm{C}$ & $\mathrm{A}$ & $\mathrm{B}$ \\
$24 / 07 / 2015$ & FRIDAY & $\mathrm{C}$ & $\mathrm{A}$ & $\mathrm{B}$ & $\mathrm{C}$ \\
$25 / 07 / 2015$ & SATURDAY & $\mathrm{A}$ & $\mathrm{B}$ & $\mathrm{C}$ & $\mathrm{A}$ \\
$26 / 07 / 2015$ & SUNDAY & $\mathrm{B}$ & $\mathrm{C}$ & $\mathrm{A}$ & $\mathrm{B}$ \\
$27 / 07 / 2015$ & MONDAY & $\mathrm{C}$ & $\mathrm{A}$ & $\mathrm{B}$ & $\mathrm{C}$ \\
$28 / 07 / 2015$ & TUESDAY & $\mathrm{A}$ & $\mathrm{B}$ & $\mathrm{C}$ & $\mathrm{A}$ \\
$29 / 07 / 2015$ & WEDNESDAY & $\mathrm{B}$ & $\mathrm{C}$ & $\mathrm{A}$ & $\mathrm{B}$ \\
$30 / 07 / 2015$ & THURSDAY & $\mathrm{C}$ & $\mathrm{A}$ & $\mathrm{B}$ & $\mathrm{C}$ \\
$31 / 07 / 2015$ & FRIDAY & $\mathrm{A}$ & $\mathrm{B}$ & $\mathrm{C}$ & $\mathrm{A}$ \\
\hline
\end{tabular}

$\mathrm{A}=$ Agbarho/Eku, $\mathrm{B}=$ Aladja, $\mathrm{C}=\mathrm{Isoko} /$ Kwale

Table 5 Load shedding rosters in August, 2015 at 60MVA, 132KV/33KV transformer T1

\begin{tabular}{|c|c|c|c|c|c|}
\hline \multirow[t]{2}{*}{ Date } & \multirow[t]{2}{*}{ Day } & \multicolumn{4}{|c|}{ Period of the day } \\
\hline & & 06:00-10:00 & 10:00-02:00 & 02:00-06:00 & 06:00-06:00 \\
\hline $01 / 08 / 2015$ & SATURDAY & B & $\mathrm{C}$ & A & B \\
\hline $02 / 08 / 2015$ & MONDAY & $\mathrm{C}$ & A & B & $\mathrm{C}$ \\
\hline 03/08/2015 & THURSDAY & A & B & $\mathrm{C}$ & A \\
\hline 04/08/2015 & FRIDAY & B & $\mathrm{C}$ & A & B \\
\hline 05/08/2015 & SATURDAY & $\mathrm{C}$ & A & B & $\mathrm{C}$ \\
\hline 06/08/2015 & SUNDAY & A & B & $\mathrm{C}$ & A \\
\hline 07/08/2015 & MONDAY & B & $\mathrm{C}$ & A & B \\
\hline 08/08/2015 & TUESDAY & $\mathrm{C}$ & A & B & $\mathrm{C}$ \\
\hline 09/08/2015 & WEDNESDAY & A & B & $\mathrm{C}$ & A \\
\hline 10/08/2015 & THURSDAY & B & $\mathrm{C}$ & A & B \\
\hline 11/08/2015 & FRIDAY & $\mathrm{C}$ & A & B & $\mathrm{C}$ \\
\hline 12/08/2015 & SATURDAY & A & B & $\mathrm{C}$ & A \\
\hline 13/08/2015 & SUNDAY & B & $\mathrm{C}$ & A & B \\
\hline 14/08/2015 & MONDAY & $\mathrm{C}$ & A & B & $\mathrm{C}$ \\
\hline 15/08/2015 & TUESDAY & A & B & $\mathrm{C}$ & A \\
\hline $16 / 08 / 2015$ & WEDNESDAY & B & $\mathrm{C}$ & A & B \\
\hline 17/08/2015 & THURSDAY & $\mathrm{C}$ & A & B & $\mathrm{C}$ \\
\hline 18/08/2015 & FRIDAY & A & B & $\mathrm{C}$ & A \\
\hline 19/08/2015 & SATURDAY & B & $\mathrm{C}$ & A & B \\
\hline 20/08/2015 & SUNDAY & $\mathrm{C}$ & A & B & $\mathrm{C}$ \\
\hline 21/08/2015 & MONDAY & A & B & $\mathrm{C}$ & A \\
\hline 22/08/2015 & TUESDAY & B & $\mathrm{C}$ & A & B \\
\hline 23/08/2015 & WEDNESDAY & $\mathrm{C}$ & A & B & $\mathrm{C}$ \\
\hline 24/08/2015 & THURSDAY & A & B & $\mathrm{C}$ & A \\
\hline 25/08/2015 & FRIDAY & B & $\mathrm{C}$ & A & B \\
\hline 26/08/2015 & SATURDAY & $\mathrm{C}$ & A & B & $\mathrm{C}$ \\
\hline 27/08/2015 & SUNDAY & A & B & $\mathrm{C}$ & A \\
\hline 28/08/2015 & MONDAY & B & $\mathrm{C}$ & A & B \\
\hline 29/08/2015 & TUESDAY & $\mathrm{C}$ & A & B & $\mathrm{C}$ \\
\hline 30/08/2015 & WEDNESDAY & A & B & $\mathrm{C}$ & A \\
\hline $31 / 08 / 2015$ & THURSDAY & $\mathrm{B}$ & $\mathrm{C}$ & A & B \\
\hline
\end{tabular}

$\mathrm{A}=$ Agbarho/Eku, B=Aladja, $\mathrm{C}=\mathrm{Isoko} / \mathrm{Kwale}$

3.Results and discussion

3.1Results of hour-meter, voltage and current measurements
The results of the hour-meter, voltage and current measurements for the months of June, July and August, 2015 at two different distribution substations $(\mathrm{S} / \mathrm{S})$ in Oleh community are presented in Tables 6, 7, 8, 9, 10 and 11 respectively. 
Table 6 Hour meter reading in the month of June 2015 at Emiye S/S

\begin{tabular}{|c|c|c|c|c|c|}
\hline \multirow[t]{2}{*}{ Days } & \multicolumn{3}{|c|}{ Time } & \multirow{2}{*}{$\begin{array}{c}\text { Voltages } \\
\text { (V) }\end{array}$} & \multirow{2}{*}{$\begin{array}{c}\text { Current } \\
\text { (I) }\end{array}$} \\
\hline & $\begin{array}{l}\operatorname{Previous} \\
\text { time }\left(C_{t}\right)\end{array}$ & $\begin{array}{c}\text { Present } \\
\text { time }\left(P_{t}\right)\end{array}$ & $\operatorname{Actual}$ time $\left(A_{t}\right)$ & & \\
\hline 1 & 11:01 & 11:01 & - & - & - \\
\hline 2 & 11:01 & 11:01 & - & - & - \\
\hline 3 & $11: 01$ & $16: 46$ & $5 \mathrm{hr} / 45 \mathrm{mins}$ & 208 & 0.98 \\
\hline 4 & $16: 46$ & 20:00 & $3 \mathrm{hrs} / 14 \mathrm{mins}$ & 208 & 0.98 \\
\hline 5 & 20:00 & 20:00 & - & - & - \\
\hline 6 & $20: 00$ & $20: 10$ & $10 \mathrm{mins}$ & 206 & 0.99 \\
\hline 7 & $20: 10$ & $20: 10$ & - & - & - \\
\hline 8 & $20: 10$ & $20: 10$ & - & - & - \\
\hline 9 & $20: 10$ & $29: 22$ & $9 \mathrm{hrs} / 12 \mathrm{mins}$ & 208 & 0.98 \\
\hline 10 & $29: 22$ & $29: 22$ & - & - & - \\
\hline 11 & $29: 22$ & $30: 18$ & $56 \mathrm{mins}$ & 210 & 0.97 \\
\hline 12 & $30: 18$ & $33: 40$ & $3 \mathrm{hrs} / 22 \mathrm{mins}$ & 208 & 0.98 \\
\hline 13 & $33: 40$ & $33: 40$ & - & - & - \\
\hline 14 & $33: 40$ & $33: 40$ & - & - & - \\
\hline 15 & $33: 40$ & $33: 40$ & - & - & - \\
\hline 16 & $33: 40$ & $33: 40$ & - & - & - \\
\hline 17 & $33: 40$ & $44: 31$ & $10 \mathrm{hrs} / 51 \mathrm{mins}$ & 210 & 0.97 \\
\hline 18 & $44: 31$ & $44: 31$ & - & - & - \\
\hline 19 & $44: 31$ & $44: 31$ & - & - & - \\
\hline 20 & $44: 31$ & $44: 31$ & - & - & - \\
\hline 21 & $44: 31$ & $52: 49$ & $8 \mathrm{hrs} / 18 \mathrm{mins}$ & 208 & 0.98 \\
\hline 22 & $52: 49$ & $61: 24$ & $8 \mathrm{hrs} / 35 \mathrm{mins}$ & 205 & 0.99 \\
\hline 23 & $61: 24$ & $61: 24$ & - & - & - \\
\hline 24 & $61: 24$ & $61: 24$ & - & - & - \\
\hline 25 & $61: 24$ & $61: 24$ & - & - & - \\
\hline 26 & $61: 24$ & $61: 24$ & - & - & - \\
\hline 27 & $61: 24$ & $70: 01$ & $9 \mathrm{hrs} / 17 \mathrm{mins}$ & 207 & 0.98 \\
\hline 28 & $70: 01$ & $70: 01$ & - & - & - \\
\hline 29 & $70: 01$ & $75: 09$ & $5 \mathrm{hrs} / 8 \mathrm{mins}$ & 208 & 0.98 \\
\hline 30 & 75:09 & $86: 07$ & $10 \mathrm{hrs} / 58 \mathrm{mins}$ & 207 & 0.98 \\
\hline
\end{tabular}

Table 7 Hour meter reading in the month of July 2015 at Emiye S/S

\begin{tabular}{|c|c|c|c|c|c|}
\hline \multirow[t]{2}{*}{ Days } & \multicolumn{3}{|c|}{ Time } & \multirow{2}{*}{$\begin{array}{c}\text { Voltages } \\
\text { (V) }\end{array}$} & \multirow{2}{*}{$\begin{array}{c}\text { Current } \\
\text { (I) }\end{array}$} \\
\hline & $\begin{array}{l}\text { Previous } \\
\text { time }\left(C_{t}\right)\end{array}$ & $\begin{array}{c}\text { Present } \\
\text { time }\left(P_{t}\right)\end{array}$ & $\operatorname{Actual} \operatorname{time}\left(A_{t}\right)$ & & \\
\hline 1 & $86: 07$ & $90: 49$ & $4 \mathrm{hrs} / 42 \mathrm{mins}$ & 208 & 0.98 \\
\hline 2 & $90: 49$ & $98: 41$ & $8 \mathrm{hrs} / 32 \mathrm{mins}$ & 208 & 0.98 \\
\hline 3 & $98: 41$ & $98: 41$ & - & - & - \\
\hline 4 & $98: 41$ & $98: 41$ & - & - & - \\
\hline 5 & $98: 41$ & $98: 41$ & - & - & - \\
\hline 6 & $98: 41$ & 109:20 & $11 \mathrm{hrs} / 19 \mathrm{mins}$ & 208 & 0.98 \\
\hline 7 & $109: 20$ & 118:03 & $9 \mathrm{hrs} / 23 \mathrm{mins}$ & 210 & \\
\hline 8 & 118:03 & 118:03 & - & - & - \\
\hline 9 & 118:03 & 118:03 & - & - & - \\
\hline 10 & 118:03 & 118:03 & - & - & - \\
\hline 11 & 118:03 & 123:09 & $5 \mathrm{hrs} / 06 \mathrm{mins}$ & 208 & 0.98 \\
\hline 12 & 123:09 & $131: 47$ & $8 \mathrm{hrs} / 38 \mathrm{mins}$ & 208 & 0.98 \\
\hline 13 & $131: 47$ & $131: 47$ & - & - & - \\
\hline 14 & 131:47 & $142: 51$ & $11 \mathrm{hrs} / 04 \mathrm{mins}$ & 207 & 0.98 \\
\hline 15 & $142: 51$ & $142: 51$ & - & - & - \\
\hline 16 & $142: 51$ & $142: 51$ & - & - & - \\
\hline 17 & $142: 51$ & $147: 56$ & $5 \mathrm{hrs} / 05 \mathrm{mins}$ & 208 & 0.98 \\
\hline 18 & $147: 51$ & $147: 51$ & - & - & - \\
\hline 19 & 147:51 & 147:51 & - & - & - \\
\hline 20 & 147:51 & $147: 51$ & - & - & - \\
\hline
\end{tabular}


International Journal of Advanced Computer Research, Vol 6(23)

\begin{tabular}{lllccc}
\hline 21 & $147: 51$ & $147: 51$ & - & - & - \\
22 & $147: 51$ & $147: 51$ & - & 208 & - \\
23 & $147: 51$ & $155: 59$ & $8 \mathrm{hrs} / 08 \mathrm{mins}$ & 207 & 0.98 \\
24 & $155: 59$ & $159: 11$ & $3 \mathrm{hrs} / 52 \mathrm{mins}$ & - & - \\
25 & $159: 11$ & $159: 11$ & - & - & - \\
26 & $159: 11$ & $159: 11$ & - & 208 & - \\
27 & $159: 11$ & $159: 11$ & - & 208 & 0.98 \\
28 & $159: 11$ & $160: 23$ & $1 \mathrm{hr} / 12 \mathrm{mins}$ & 208 & 0.98 \\
29 & $160: 23$ & $167: 00$ & $7 \mathrm{hrs} / 17 \mathrm{mins}$ & 208 & 0.98 \\
30 & $167: 00$ & $175: 17$ & $6 \mathrm{hrs} / 17 \mathrm{mins}$ & 68 \\
31 & $175: 17$ & $181: 17$ & $6 \mathrm{hrs}$ & & 0.98 \\
\hline
\end{tabular}

Table 8 Hour meter reading in the month of August 2015 at Emiye S/S

\begin{tabular}{|c|c|c|c|c|c|}
\hline \multirow[t]{2}{*}{ Days } & \multicolumn{3}{|c|}{ Time } & \multirow{2}{*}{$\begin{array}{c}\text { Voltages } \\
\text { (V) }\end{array}$} & \multirow{2}{*}{$\begin{array}{c}\text { Current } \\
\text { (I) }\end{array}$} \\
\hline & $\begin{array}{l}\text { Previous } \\
\text { time }\left(C_{t}\right)\end{array}$ & $\operatorname{Present}$ time $\left(\boldsymbol{P}_{t}\right)$ & $\operatorname{Actual}$ time $\left(\boldsymbol{A}_{\boldsymbol{t}}\right)$ & & \\
\hline 1 & $181: 17$ & $181: 17$ & - & - & - \\
\hline 2 & $181: 17$ & 181:17 & - & - & - \\
\hline 3 & $181: 17$ & $181: 17$ & - & - & - \\
\hline 4 & $181: 17$ & $181: 51$ & $34 \mathrm{mins}$ & 208 & 0.98 \\
\hline 5 & 181:51 & 181:51 & - & - & - \\
\hline 6 & $181: 51$ & $189: 33$ & $8 \mathrm{hrs} / 23 \mathrm{mins}$ & 209 & 0.97 \\
\hline 7 & $189: 33$ & 198:06 & $9 \mathrm{hrs} / 13 \mathrm{mins}$ & 208 & 0.98 \\
\hline 8 & 198:06 & 205:22 & $7 \mathrm{hrs} / 16 \mathrm{mins}$ & 208 & \\
\hline 9 & $205: 22$ & $205: 22$ & - & - & - \\
\hline 10 & $205: 22$ & 214:05 & $9 \mathrm{hrs} / 23 \mathrm{mins}$ & 207 & 0.98 \\
\hline 11 & 214:05 & 218:07 & $4 \mathrm{hrs} / 02 \mathrm{mins}$ & 208 & 0.98 \\
\hline 12 & 218:07 & 218:07 & - & - & - \\
\hline 13 & 218:07 & 218:07 & - & - & - \\
\hline 14 & 218:07 & 227:39 & $9 \mathrm{hrs} / 32 \mathrm{mins}$ & 207 & 0.98 \\
\hline 15 & $227: 39$ & $230: 40$ & $3 \mathrm{hrs} / 01 \mathrm{~min}$ & 208 & 0.98 \\
\hline 16 & $230: 40$ & $230: 40$ & - & - & - \\
\hline 17 & $230: 40$ & 231:03 & $1 \mathrm{hr} / 03 \mathrm{mins}$ & 208 & 0.98 \\
\hline 18 & 231:03 & 231:03 & - & - & - \\
\hline 19 & 231:03 & 231:03 & - & - & - \\
\hline 20 & 231:03 & 239:17 & $8 \mathrm{hrs} / 14 \mathrm{mins}$ & 208 & 0.98 \\
\hline 21 & 239:17 & 239:57 & 40mins & 208 & 0.98 \\
\hline 22 & 239:57 & 239:57 & - & - & - \\
\hline 23 & $239: 57$ & 239:57 & - & - & - \\
\hline 24 & 239:57 & 248:06 & $8 \mathrm{hrs} / 49 \mathrm{mins}$ & 208 & 0.98 \\
\hline 25 & 248:06 & 248:06 & - & - & - \\
\hline 26 & 248:06 & $252: 16$ & $4 \mathrm{hrs} / 10 \mathrm{mins}$ & 208 & 0.98 \\
\hline 27 & $252: 16$ & $252: 16$ & - & - & - \\
\hline 28 & $256: 16$ & 267:02 & $11 \mathrm{hrs} / 26 \mathrm{mins}$ & 207 & 0.98 \\
\hline 29 & 267:02 & 267:02 & - & - & - \\
\hline 30 & $267: 02$ & 278:04 & $11 \mathrm{hrs} / 02 \mathrm{mins}$ & 208 & 0.98 \\
\hline 31 & 278:04 & 287:51 & $9 \mathrm{hrs} / 47 \mathrm{mins}$ & 208 & 0.98 \\
\hline
\end{tabular}

Table 9 Hour meter reading in the month of June 2015 at Campus S/S

\begin{tabular}{|c|c|c|c|c|c|}
\hline \multirow[t]{2}{*}{ Days } & \multicolumn{3}{|c|}{ Time } & \multirow{2}{*}{$\begin{array}{c}\text { Voltages } \\
\text { (V) }\end{array}$} & \multirow{2}{*}{$\begin{array}{l}\text { Current } \\
\text { (I) }\end{array}$} \\
\hline & $\begin{array}{l}\text { Previous } \\
\text { time }\left(C_{t}\right)\end{array}$ & $\operatorname{Present}$ time $\left(\boldsymbol{P}_{\boldsymbol{t}}\right)$ & $\operatorname{Actual}$ time $\left(\boldsymbol{A}_{t}\right)$ & & \\
\hline 1 & 00:04 & $05: 06$ & $5 \mathrm{hrs} / 02 \mathrm{mins}$ & 208 & 0.98 \\
\hline 2 & $05: 06$ & 07:08 & $2 \mathrm{hrs} / 02 \mathrm{mins}$ & 208 & 0.98 \\
\hline 3 & 07:08 & 07:08 & - & - & - \\
\hline 4 & 07:08 & 13:09 & $6 \mathrm{hrs} / 01 \mathrm{~min}$ & 207 & 0.98 \\
\hline 5 & 13:09 & 13:09 & - & - & - \\
\hline 6 & 13:09 & 13:09 & - & - & - \\
\hline
\end{tabular}




\begin{tabular}{|c|c|c|c|c|c|}
\hline \multirow[t]{2}{*}{ Days } & \multicolumn{3}{|c|}{ Time } & \multirow{2}{*}{$\begin{array}{c}\text { Voltages } \\
\text { (V) }\end{array}$} & \multirow{2}{*}{$\begin{array}{c}\text { Current } \\
\text { (I) }\end{array}$} \\
\hline & $\begin{array}{l}\text { Previous } \\
\text { time }\left(C_{t}\right)\end{array}$ & Present time $\left(P_{t}\right)$ & $\operatorname{Actual}$ time $\left(\boldsymbol{A}_{t}\right)$ & & \\
\hline 7 & $13: 09$ & $26: 06$ & $12 \mathrm{hrs} / 57 \mathrm{mins}$ & 208 & 0.98 \\
\hline 8 & 26:06 & $26: 06$ & - & - & - \\
\hline 9 & $26: 06$ & $26: 06$ & - & - & - \\
\hline 10 & $26: 06$ & $26: 06$ & - & - & - \\
\hline 11 & $26: 06$ & 30:09 & $4 \mathrm{hrs} / 03 \mathrm{mins}$ & 209 & 0.97 \\
\hline 12 & 30:09 & 31:09 & $1 \mathrm{hr}$ & 209 & \\
\hline 13 & $31: 09$ & 31:09 & - & - & - \\
\hline 14 & 31:09 & 31:09 & - & - & - \\
\hline 15 & $31: 09$ & $49: 33$ & $18 \mathrm{hrs} / 24 \mathrm{mins}$ & 208 & 0.98 \\
\hline 16 & $49: 33$ & $49: 33$ & - & - & - \\
\hline 17 & $49: 33$ & $53: 31$ & $3 \mathrm{hrs} / 58 \mathrm{mins}$ & 207 & 0.98 \\
\hline 18 & $53: 31$ & $53: 31$ & - & - & - \\
\hline 19 & $53: 31$ & $57: 50$ & $4 \mathrm{hrs} / 19 \mathrm{mins}$ & 208 & 0.98 \\
\hline 20 & $57: 50$ & $57: 50$ & - & - & - \\
\hline 21 & $57: 50$ & $62: 58$ & $5 \mathrm{hrs} / 08 \mathrm{mins}$ & 206 & 0.99 \\
\hline 22 & $62: 58$ & $62: 58$ & - & - & - \\
\hline 23 & $62: 58$ & $62: 58$ & - & - & - \\
\hline 24 & $62: 58$ & $78: 10$ & $15 \mathrm{hrs} / 12 \mathrm{mins}$ & 208 & 0.98 \\
\hline 25 & $78: 10$ & $80: 42$ & $2 \mathrm{hrs} / 32 \mathrm{mins}$ & 208 & 0.98 \\
\hline 26 & $80: 42$ & $80: 42$ & - & - & - \\
\hline 27 & $80: 42$ & $80: 42$ & - & - & - \\
\hline 28 & $80: 42$ & $81: 00$ & $18 \mathrm{mins}$ & 205 & 0.99 \\
\hline 29 & $81: 00$ & $92: 17$ & $11 \mathrm{hrs} / 17 \mathrm{mins}$ & 207 & 0.98 \\
\hline 30 & $92: 17$ & $98: 11$ & $5 \mathrm{hrs} / 54 \mathrm{mins}$ & 209 & 0.97 \\
\hline 31 & $98: 11$ & $105: 45$ & $7 \mathrm{hrs} / 34 \mathrm{mins}$ & 209 & 0.97 \\
\hline
\end{tabular}

Table 10 Hour meter reading in the month of July 2015 at Campus S/S

\begin{tabular}{|c|c|c|c|c|c|}
\hline \multirow[t]{2}{*}{ Days } & \multicolumn{3}{|c|}{ Time } & \multirow{2}{*}{$\begin{array}{c}\text { Voltages } \\
\text { (V) }\end{array}$} & \multirow{2}{*}{$\begin{array}{c}\text { Current } \\
\text { (I) }\end{array}$} \\
\hline & $\begin{array}{c}\text { Previous } \\
\text { Time }\left(C_{t}\right)\end{array}$ & $\begin{array}{c}\text { Present } \\
\operatorname{Time}\left(\boldsymbol{P}_{t}\right)\end{array}$ & Actual Time $\left(A_{t}\right)$ & & \\
\hline 1 & $105: 45$ & 109:20 & $3 \mathrm{hrs} / 35 \mathrm{mins}$ & 208 & 0.98 \\
\hline 2 & 109:20 & 113:30 & $4 \mathrm{hrs} / 10 \mathrm{mins}$ & 208 & 0.98 \\
\hline 3 & 113:30 & $116: 00$ & $2 \mathrm{hrs} / 30 \mathrm{mins}$ & 205 & \\
\hline 4 & 116:00 & 116:00 & - & - & - \\
\hline 5 & 116:00 & 116:00 & - & - & - \\
\hline 6 & $116: 00$ & $116: 30$ & $30 \mathrm{mins}$ & 225 & 0.91 \\
\hline 7 & - & - & - & - & - \\
\hline 8 & - & - & - & - & - \\
\hline 9 & - & - & - & - & - \\
\hline 10 & - & - & - & - & - \\
\hline 11 & - & - & - & - & - \\
\hline 12 & 00:03 & 05:52 & $5 \mathrm{hrs} / 49 \mathrm{mins}$ & 203 & 1.00 \\
\hline 13 & 05:52 & 05:52 & - & - & - \\
\hline 14 & 05:52 & 09:07 & $3 \mathrm{hrs} / 15 \mathrm{mins}$ & 207 & 0.98 \\
\hline 15 & 09:07 & 09:07 & - & - & - \\
\hline 16 & 09:07 & 09:07 & - & - & - \\
\hline 17 & 09:07 & 09:07 & - & - & - \\
\hline 18 & 09:07 & $17: 02$ & $7 \mathrm{hrs} / 55 \mathrm{mins}$ & 204 & 0.99 \\
\hline 19 & $17: 02$ & $17: 02$ & - & - & - \\
\hline 20 & $17: 02$ & $19: 22$ & $2 \mathrm{hrs} / 20 \mathrm{mins}$ & 208 & 0.98 \\
\hline 21 & $19: 22$ & $19: 22$ & - & - & - \\
\hline 22 & $19: 22$ & $23: 22$ & $4 \mathrm{hrs}$ & 205 & 0.99 \\
\hline 23 & $23: 22$ & $23: 22$ & - & - & - \\
\hline 24 & $23: 22$ & $23: 22$ & - & - & - \\
\hline 25 & $23: 22$ & $23: 22$ & - & - & - \\
\hline
\end{tabular}


International Journal of Advanced Computer Research, Vol 6(23)

\begin{tabular}{|c|c|c|c|c|c|}
\hline \multirow[t]{2}{*}{ Days } & \multicolumn{3}{|c|}{ Time } & \multirow{2}{*}{$\begin{array}{c}\text { Voltages } \\
\text { (V) }\end{array}$} & \multirow{2}{*}{$\begin{array}{c}\text { Current } \\
\text { (I) }\end{array}$} \\
\hline & $\begin{array}{c}\text { Previous } \\
\text { Time }\left(C_{t}\right)\end{array}$ & $\begin{array}{c}\text { Present } \\
\operatorname{Time}\left(P_{t}\right)\end{array}$ & $\operatorname{Actual} \operatorname{Time}\left(A_{t}\right)$ & & \\
\hline 26 & $23: 22$ & $26: 47$ & $3 \mathrm{hrs} / 25 \mathrm{mins}$ & 203 & 1.00 \\
\hline 27 & $26: 47$ & $26: 47$ & - & - & - \\
\hline 28 & $26: 47$ & 29:02 & $2 \mathrm{hrs} / 15 \mathrm{mins}$ & 207 & 0.98 \\
\hline 29 & 29:02 & 29:02 & - & - & - \\
\hline 30 & 29:02 & $37: 11$ & $8 \mathrm{hrs} / 9 \mathrm{mins}$ & 205 & 0.99 \\
\hline 31 & $37: 11$ & $44: 41$ & $7 \mathrm{hrs} / 30 \mathrm{mins}$ & 208 & 0.98 \\
\hline
\end{tabular}

Table 11 Hour meter reading in the month of August 2015 at Campus S/S

\begin{tabular}{|c|c|c|c|c|c|}
\hline \multirow[t]{2}{*}{ Days } & \multicolumn{3}{|c|}{ Time } & \multirow{2}{*}{$\begin{array}{c}\text { Voltages } \\
\text { (V) }\end{array}$} & \multirow{2}{*}{$\begin{array}{l}\text { Curren } \\
\text { (I) }\end{array}$} \\
\hline & $\begin{array}{l}\text { Previous } \\
\text { Time }\left(C_{t}\right)\end{array}$ & $\begin{array}{c}\text { Present } \\
\operatorname{Time}\left(\boldsymbol{P}_{t}\right)\end{array}$ & $\operatorname{Actual} \operatorname{Time}\left(\boldsymbol{A}_{t}\right)$ & & \\
\hline 1 & $44: 41$ & $45: 42$ & $1 \mathrm{hr} / 1 \mathrm{~min}$ & 203 & 1.00 \\
\hline 2 & $45: 42$ & $45: 42$ & - & - & - \\
\hline 3 & $45: 42$ & $45: 42$ & - & - & - \\
\hline 4 & $45: 42$ & $48: 46$ & $3 \mathrm{hrs} / 4 \mathrm{mins}$ & 205 & 0.99 \\
\hline 5 & $48: 46$ & $51: 54$ & $3 \mathrm{hrs} / 8 \mathrm{mins}$ & 205 & 0.99 \\
\hline 6 & $51: 54$ & $51: 54$ & - & - & - \\
\hline 7 & $51: 54$ & $56: 59$ & $5 \mathrm{hrs} / 5 \mathrm{mins}$ & 203 & 1.00 \\
\hline 8 & $56: 59$ & $56: 59$ & - & - & - \\
\hline 9 & $56: 59$ & 61:02 & $4 \mathrm{hrs} / 3 \mathrm{mins}$ & 208 & 0.98 \\
\hline 10 & 61:02 & $63: 05$ & $2 \mathrm{hrs} / 3 \mathrm{mins}$ & 205 & 0.99 \\
\hline 11 & $63: 05$ & $63: 05$ & - & - & - \\
\hline 12 & $63: 05$ & $63: 05$ & - & - & - \\
\hline 13 & $63: 05$ & $63: 05$ & - & - & - \\
\hline 14 & $63: 05$ & $67: 07$ & $4 \mathrm{hrs} / 2 \mathrm{mins}$ & 208 & 0.98 \\
\hline 15 & $67: 07$ & $67: 07$ & - & - & - \\
\hline 16 & $67: 07$ & 78:08 & $11 \mathrm{hrs} / 1 \mathrm{~min}$ & 209 & 0.97 \\
\hline 17 & 78:08 & 78:08 & - & - & - \\
\hline 18 & 78:08 & $84: 12$ & $6 \mathrm{hrs} / 4 \mathrm{mins}$ & 207 & 0.98 \\
\hline 19 & $84: 12$ & $84: 12$ & - & - & - \\
\hline 20 & $84: 12$ & $84: 12$ & - & - & - \\
\hline 21 & $84: 12$ & $84: 12$ & - & - & - \\
\hline 22 & $84: 12$ & $84: 12$ & - & - & - \\
\hline 23 & $84: 12$ & $84: 12$ & - & - & - \\
\hline 24 & $84: 12$ & $92: 14$ & $8 \mathrm{hrs} / 2 \mathrm{mins}$ & 208 & 0.98 \\
\hline 25 & $92: 14$ & $92: 14$ & - & - & - \\
\hline 26 & $92: 14$ & $92: 14$ & - & - & - \\
\hline 27 & $92: 14$ & $92: 14$ & - & - & - \\
\hline 28 & $92: 14$ & $107: 16$ & $15 \mathrm{hrs} / 2 \mathrm{mins}$ & 209 & 0.97 \\
\hline 29 & $107: 16$ & $114: 21$ & $7 \mathrm{hrs} / 5 \mathrm{mins}$ & 207 & 0.98 \\
\hline 30 & $114: 21$ & $125: 24$ & $11 \mathrm{hrs} / 3 \mathrm{mins}$ & & \\
\hline 31 & $125: 24$ & $125: 24$ & - & - & - \\
\hline
\end{tabular}

3.2Discussion of results

The data presented in Tables 6, 7, 8, 9, 10 and 11 were used to determine the percentage mean hour of power availability for the period of three months.

Equation 4 was used to calculate the percentage mean hour for the month at the two sub-stations.

$$
\boldsymbol{H}_{m}=\sum \frac{\boldsymbol{A}_{t}}{\boldsymbol{H}}
$$

Where $\mathrm{H}_{\mathrm{m}}$ is mean hour of supply

$A_{t}$ is Actual read hour from hour meter
$\mathrm{H}_{\mathrm{d}}$ is number of hours in a month For the month of June at the two different substations, the percentage mean hour is deduced as follows:

From Table 6,

$$
\begin{aligned}
& \frac{\sum A_{t}}{\sum H_{d}} \\
& \begin{array}{r}
5.45+3.14+0.10+9.12+0.56+3.22+10.51+ \\
8.18+8.35+9.17+5.8+10.58
\end{array} \\
& =\frac{24 \times 30}{24}
\end{aligned}
$$


S.O.Otuagoma

$$
=\frac{70 h r s / 357 \text { mins }}{720 h r s}=\frac{70+\left[\frac{357}{60}\right]}{720}=0.1055
$$

From Table 9

$$
\begin{aligned}
& \frac{\sum A_{t}}{\sum H_{d}} \\
& 5.02+2.02+6.01+12.57+4.03+1.0+18.24+ \\
& 3.58+4.19+5.08+15.12+2.32+0.18+5.54+ \\
& =\frac{11.17+7.34}{24 \times 30} \\
& \quad=\frac{100 \text { hrs } / 341 \text { mins }}{720 \text { hrs }}=\frac{100+\left[\frac{341}{60}\right]}{720}=0.1468 \\
& \therefore \text { mean supply period for June } \\
& \quad=\frac{0.1055+0.1468}{2}=0.12615 \\
& =12.6 \%
\end{aligned}
$$

This $12.6 \%$ of power available for the month of June accounts for only 3 hours of power supply per day as against the load shedding roster for the month of June as presented in Table 3 where availability is supposed to be 4 hours per day. Even at that, the supply is not evenly distributed rather there are some days that there is no power supply at all as can be seen in Tables 6-11. These deductions are true for the months of July and August as well. For the fact that $87.4 \%$ of the time power are unavailable accounts for the high unreliability of electric power supply to this community.

The term reliability is closely related to outages, interruptions, failures and availability, and it is associated with switch gear, protection and control equipment, $100 \%$ reliability and availability in the power system cannot be guaranteed but high reliability of $99.8 \%$ is possible [10]. Reliability describes the ability of continuous service without outages/failures or interruptions and is expressed as [11]:

Reliability index $\beta r=$

Total Service houre-Interruption hours/yr

Total Service hours

Using equation 5 to evaluate the reliability of power supply to Oleh for the months of June - August as can be seen from Tables 6 and 9, 7 and 10, 8 and 11, From Tables 6 and 9, the mean total service hour is;

$$
\begin{gathered}
\frac{70+\left[\frac{357}{60}\right]+100+\left[\frac{341}{60}\right]}{2}=90.68 \text { hours } \\
\text { Total interuption hour }=720-90.68 \\
=629.32 \text { hours }
\end{gathered}
$$

$$
\therefore \quad \beta r=\frac{90.68-629.32}{90.68}=-5.94
$$

From Tables 7 and 10, the mean total service hour is;

$$
\begin{gathered}
\frac{92+\left[\frac{275}{60}\right]+50+\left[\frac{296}{60}\right]}{2}=75.75 \text { hours } \\
\text { Total interuption hour }=744-75.75 \\
=668.25 \text { hours } \\
\therefore \quad \beta r=\frac{75.75-668.25}{75.75}=-7.82
\end{gathered}
$$

From Tables 8 and 11, the mean total service hour is;

$$
\frac{101+\left[\frac{335}{60}\right]+80+\left[\frac{43}{60}\right]}{2}=93.65 \text { hours }
$$

Total interuption hour $=744-93.65=650.35$

$$
\therefore \quad \beta r=\frac{93.65-650.35}{93.65}=-5.94
$$

From the above results, the Oleh community is suffering greatly from unavailability of supply since the system is highly unreliable as can be seen from the negative reliability index. In-spite of this high unreliability, the community is overcharged for electricity consumption. Since most of the customers are not metered and those with meters, their meters are not read by the energy provider. Monthly an estimated bill is usually sent to the consumer whether there was supply or not. Take a case study of a customer connected to the Emiye sub-station, his estimated bill for the month of July was N2, 300 . From the hour-meter reading for that month as can be seen in Tables 7 and 10, the average hour for power availability as read from the hour meter was 96 hours on average voltage of $208 \mathrm{~V}$ and current of $0.98 \mathrm{~A}$.

Now the expected energy consumption for the month of July using equations 2 and 3 is;

$$
E=I V \operatorname{Cos} \phi t \phi_{d} \phi_{a}
$$

Where $\mathrm{E}$ is units of energy consumed in $\mathrm{kWh}$

I is supply current in Ampere, A

$\mathrm{V}$ is supply voltage in Volts, $\mathrm{V}$

Cosø is power factor, 0.85

$\mathrm{t}$ is time supply is available in hours

$\varphi_{\mathrm{d}}$ is diversity factor

$\varphi_{\mathrm{a}}$ is load factor.

Hence $\mathrm{E}=0.98 \times 208 \times 0.85 \times 96 \times 0.6 \times 0.4=$ $3.99 \mathrm{kWh}$. The electric tariff for this customer is 
N14.82k per unit + N750 fixed charge. The actual billing, therefore, is $3.99 \times 14.82=\mathrm{N} 59.13+\mathrm{N} 750=$ N809.13K. This also illustrates the fact that the consumer is not only suffering from poor power supply, he is also forced to pay for what was not consumed.

\section{Conclusion}

This study presents an assessment of the electric power availability in Oleh community using simple instruments like the hour-meter for the measurement of the number of hours' supply was available in the town. The impact of load shedding feels more in the rural areas since energy providers preferentially shed the least important load on the system during system imbalance. The technique adopted in this study for the evaluation of electricity billing will be helpful to consumers to have an informed idea of how much they are supposed to pay for electricity consumption for any given month instead of the high estimate they received from the energy providers. Further work will examine the nature of the country's tariff system and effect of estimated billing for the consumers as well as on the staff of the energy provider. Since the electric power supply industry comprising of generation, transmission and distribution system as well as utilization and marketing is described as the greatest machine created by man, its continued good performance as a part of the inevitable energy requirement is absolutely vital to the success of the nation's economy especially in the rural areas. So actions should be taken at the national, regional and international levels to accelerate the production of more electric power to satisfy the ever increasing need for this commodity.

\section{Acknowledgment}

None.

\section{Conflicts of interest}

The author has no conflicts of interest to declare.

\section{References}

[1] https://odinakadotnet.wordpress.com/. Accessed 09 May 2013.

[2] www.unido.org/fileadmin/user_media_upgrade/what_ we_do/Topics/Energy_access/WSHPDR_2013_Execu tive_summary.pdf. Accessed 20 June 2014.

[3] Isoun, T. T. Address by the Honourable Minister, Federal Ministry of Science and Technology at the Inauguration of the UNIDO Regional Centre for Small Hydropower, Abuja, Nigeria. Small Hydropower for Productive Use in Rural Areas. 2006.
[4] http://www.gtz.de/de/dokumente/gtz2010-en-HERAEUEI-PDF-framework-conditions-hydropower.pdf. Accessed 19 March 2013.

[5] Ogujor EA, Orobor NP. SAIDI minimization through conventional energy resources: opportunities and challenges. Journal of Economics and Engineering. 2010:54-6.

[6] Hazra J, Sinha AK. Congestion management using multi objective particle swarm optimization. IEEE Transactions on Power Systems. 2007; 22(4):1726-34.

[7] Rai S, Kumar Y, Agnihotri G. Under voltage load shedding for contingency analysis to optimize power loss and voltage stability margin. Electrical and Electronics Engineering: An International Journal. 2014; 3(4); 57-64.

[8] Bevrani H, Tikdari AG, Hiyama T. Power system load shedding: key issues and new perspectives. International Journal of Computer, Electrical, Automation, Control and Information Engineering. 2010; 4 (5): 886-91.

[9] Rekha A, Kumar RM, Ashok KN,Vanitha D. An intelligent load shedding to improve power system congestion. International Journal of Current Research. 2013; 5 (4); 973-7.

[10] Chinwuko CE, Chukwuneke JL, Okolie PC, Dara EJ. Modelling and optimization of electricity distribution planning system using dynamic programming techniques: a case of power holding company of nigeria (PHCN). International Journal of Multidisciplinary Sciences and Engineering. 3(6); 3946.

[11] Dickinson WH. Report on Reliability Survey of Industrial Plants, Part 1-Reliability of Electrical Equipment. In IEEE I \& CPS conference record. 1973.

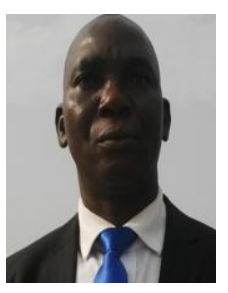

Smith Orode Otuagoma was born at Ivrogbo-Emevor in Isoko North Local Government Area of Delta State, Nigeria on the 23rd April, 1963. He received a B.Eng. degree in Electrical / Electronic Engineering at the University of Agriculture, Makurdi in 1996, an M.Eng. and Ph.D degree from the University of Benin, Edo State, Nigeria in 2000 and 2015 respectively. He is currently a lecturer at the Delta State University, Oleh Campus. His research interest is in the areas of Power Systems, Renewable Energy and Environmental Studies.

Email: otuagoma@yahoo.com 